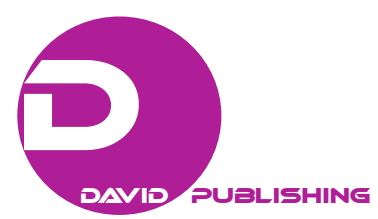

\title{
Mobile Health Applications User Trends in Turkey*
}

\author{
Seray Öney Doganyigit \\ Istanbul Bilgi University, Istanbul, Turkey \\ Elgiz Yilmaz \\ Galatasaray University, Istanbul, Turkey
}

\begin{abstract}
Due to the rapid rise of mobile phones around the globe, many consumers, researchers, clinicians, and health services are starting to see their utility in health. As well as a health informatics role in improving the uptake and efficiency of current health services, mobile communication-assisted health care (m-health) also opens opportunities for services that are strikingly new and curative, in particular delivering personal health behaviour change programmes. Herein, we report the preliminary findings of a health-promotion survey titled "Mobile Apps User Trend Analysis of Turkish People". The survey examines the information needs and media preferences of women and men who research health information and use mobile apps for their healthcare in everyday life. Also this study tries to recover the behaviour of people and what applications they are downloading with respect to health, wellness, and medical mobile applicaitons. In looking the data, it appears that adoption of mHealth is growing at a furious pace. In this context, improvements to health communication have the potential to make a significant role to a promising new medium for health education and communication.
\end{abstract}

Keywords: health communication, mobile health, mobile applications, acceptance behaviors

\section{Introduction}

New information and communications technologies (ICTs) hold promise for the creation of effective and innovative health promotion (Thackeray, Neiger, Hanson, \& McKenzie, 2008). Researchers argue that social media forms (which they define as email, texting, microblogging, and smartphone applications) can be used by health professionals to increase health engagement and improve health outcomes (Fisher \& Clayton, 2012).

In particular, Fisher and Clayton (Fisher \& Clayton, 2012) state that future studies need to consider patient's media preferences and their level of interest in the use of social media for health care. We argue that understanding users' preferences regarding different sources of health-promotion media, including ICTs, is an essential component of effective health communication (Halpert et al., 2008).

A relatively new area of interest is the use of mobile phones to deliver self contained programmes that aim to support health behavior change. Behavior modification is an important part of many public health programmes that aim to prevent some of the major global disease burdens such as cardiovascular disease, diabetes, and some cancers. Successful behavior change interventions such as smoking cessation (Rodgers et al.,

\footnotetext{
*Acknowledgment: Assistance provided by Erden Asena and his company "doktorsitesi.com", and also "tekdozdijital.com" has been a great help in collecting data. We would also like to thank Özgün Afşar in gathering data. This paper is financially supported by Galatasaray University Scientific Research Projects Commission within the project no. 14.300.003.

Seray Öney Doganyigit, lecturer, Faculty of Communication, Istanbul Bilgi University.

Elgiz Y1lmaz, associate professor, Faculty of Communication, Galatasaray University.
} 
2005) and weight management through diet or physical activity are some of them (Tate, Wing, \& Winett, 2001).

\section{The Framework of Mobile Health Information Technologies and Mobile Applications}

Mhealth is a reasonably a new term that has been defined by The Global Observatory for eHealth (GOe), as medical and public health practice supported by mobile devices, such as mobile phones, patient monitoring devices, personal digital assistants (PDAs), and other wireless devices. mHealth involves the use and capitalization on a mobile phone's core utility of voice and short messaging service (SMS) as well as more complex functionalities and applications including general packet radio service (GPRS), third and fourth generation mobile telecommunications (3G and 4G systems), global positioning system (GPS), and Bluetooth technology.

The use of mobile and wireless technologies to support the achievement of health objectives (Mhealth) has the potential to transform the face of delivery across the World (WHO). According to the International Communication Union (ITU), there are now over five billion wireless subscribers; over $70 \%$ of them reside in low-middle-income countries. The GSM association reports commercial wireless signals cover over $85 \%$ of the world's population, extending far beyond the reach of the electrical grid (WHO).

\section{Health Communication and New Information Technologies}

Health systems worldwide are under increasing pressure to perform under multiple health challenges, chronic staff shortages, and limited budgets, all of which makes choosing interventions difficult.

Health care industry is searching for a series of mHealth deployments worldwide that are providing early evidence of the potential for mobile and wireless technologies. mHealth is being applied in maternal and child health, and programmes reducing the burden of the diseases linked with poverty, including HIV/AIDS, malaria, and tuberculosis (TB) (WHO). mHealth applications are being tested in such diverse scenarios as improving timely access to emergency and general health services and information, managing patient care, reducing drug shortages at health clinics, enhancing clinical diagnosis and treatment adherence, among others.

To date medical literature includes a few studies only a small number of uses, many of which aim to make healthcare systems more efficient and improve existing health services for patients such as sending reminders of appointments to increase attendance rates (Downer, Meara, Da Costa, \& Sethuraman, 2006; Leong et al., 2006) or empowering the patient by enhancing self monitoring by providing real time feedback (Connelly et al., 2006; Klasnja et al., 2006). While some of them aim to improve care for those with chronic diseases, such as texting reminders to improve adherence to treatment in people with HIV (Puccio et al., 2006), the others aim to promote youth sexual health and disease prevention by downloading applications via smartphones (Levine, 2007). More advanced programs facilitate two-way communications between clients and their healthcare providers, for example, in the self management of diabetes, with clients texting their blood glucose levels and their clinicians reporting back recommendations to alter management (Kim, H-S., Kim, N-C., \& Ahn, 2006; Ferrer-Roca, Cardenas, \& Diaz-Cardama, 2004).

\section{Mobile Health Applications and User Trends}

Mobile phones are integrated into people's daily lives, tending to be always on and always carried, communication with participants in programmes can be regular and frequent and messages can be delivered in a time- sensitive or time appropriate manner. Participants can view messages at a time that suits them or ask for help when they actually need it. Messages of various sorts can be delivered directly to people, dealing with any 
issues of fidelity to the original intent of the programme if delivered via third party.

According to Ruder Finn report ${ }^{1}$, mobile subscribers worldwide expected to reach 6.9 billion by the end of 2013. Worldwide, we are adopting new technologies at an increasing speed with the number of smartphones in use now exceeding one billion, over a $47 \%$ increase from a year earlier. The rise in ownership is set to continue as cellular and data packages make smartphones more affordable. As smartphones become integrated into the consumers' everyday life, so does the appetite for mobile apps. Similarly, the tablet market is booming. In the U.S., nearly a quarter of adults (22\%) own a tablet device and $23 \%$ of no tablet owners plan to purchase one within the next six months. Roughly 75 million individuals (about $31 \%$ of the U.S. population) have used mobile phones for health information and apps in 2012.

However, according to ICT Q4 report (Information and Communication Technology Authorities). There are more than 69 million mobile subscribers corresponding to $90.9 \%$ penetration rate in Turkey. Mobile penetration rate exceeds $100 \%$ when $0-9$ year's old population are excluded. There are more than 11 million of these subscribers account for smartphone subscribers (IPSOS Media Ct., 2013)

The opportunities these new platforms create for healthcare services to be delivered in an entirely new way have been recognized by technology and mobile vendors. Companies are investing millions to develop new apps and services - both for the consumer and for the healthcare professional. There is no doubt that mobile apps are incredibly popular. Our survey aims to help answer questions such as "Is there a market for health apps in Turkey?" and "Do consumers want to have healthcare services on mobile devices?" in order to provide greater understanding of what kind of role mHealth plays today and what it will moving forward in the increasingly mobile world.

\section{Mobile Health Care and Users}

Mhealth is the practice of medicine and public health, supported by mobile devices. Transformative trends in the industry are conducive to remarkable growth in mobile applications-population aging, increasing chronic illness, accelerating health costs, new regulatory reforms, and increased consumer demand for health information and self-care will drive mobile solution growth.

Mobile device users who downloaded at least one mobile health application onto their smartphone doubled between 2011 and $2012^{2}$. In order to gain insight into the user trends to mobile health applications in Turkey, a survey has been performed. A selection of some preliminary results is presented here.

\section{Method}

We conducted an online survey of 25 questions. Twenty of the survey questions were closed-ended investigating socio-demographic data such as gender, age, education, occupation/job, income group, also the Internet and mobile phone usage rates, brand preferences in mobile phone; and five of them were open-ended investigating the usage rates of mobile health applications and for what purpose they are used.

The study was performed with approximate 6000 people from Turkey. Participants were recruited from April 2013 to August 2013. In order to get a good response rate, the sample was chosen randomly between "doktorsitesi.com" web site users — which is one of the most visited medical web site—in Turkey.

\footnotetext{
1 Ruder-Finn. The healthcare and technology teams commissioned research by ORC. Retrieved from http://www.ruderfinn.com/pdf/Ruder\%20Finn\%20US\%20mHealth\%20report\%20FINAL.pdf

2 research2guidance. US\$ 1.3 billion: The market for mHealth applications. in 2012. Available from http://www.research2guidance.com/us-1.3-billion-the-market-for-mhealth-applications-in-2012/
} 


\section{Findings}

Insight into health care consumers' behaviors and attitudes are critical informations in an environment where participants are active in managing in their health care. Our findings suggest that:

(1) The majority of the participants were well-educated namely, $15 \%$ were having master degree, $58 \%$ graduated degree, $23 \%$ were graduated from high school and $4 \%$ from primary school.

(2) Seventy one percent of participants use mobile smart phones. Fifty seven percent of them were women and $47 \%$ were men. Our findings suggest that the usage of mobile smart phone increases when the age and education level raise in both men and women.

Fifteen percent of mobile smartphone users (71\%) use their phones to look up health information and downloaded mHealth application. Their high possession rates, connectivity, and capabilities range make mobile technologies ideal for use in the health care industry ${ }^{3}$. Mobile devices can offer greater convenience and flexibility for both patients and providers.

(1) Ninth five percent of the participants look up health information on Internet. Fifty six percent of them were women and $44 \%$ were men. We may suggest that there is a significant relationship between the participants' age and the health information topics searched on internet by men and by women. Thus, $13 \%$ of women younger than 35 years old are the most likely to access personal health, wellness, mother healthcare before and after delivery and baby care informations, while those age $45+(15 \%)$ are least likely to access women health and chronic diseases informations. Twelve percent of men 35 years old or younger reported having looked up personal health, well-being, musculation, fitness, while those age 45+ (11\%) have done the same research as women $45+$ years old.

(2) Mobile application users who downloaded at least one mHealth app onto their smartphone were $68 \%$.

(3) Health information (primarily oriented towards personal health, wellness, fitness, and information on health services) is the fastest-growing content category for Turkish mobile users, in both women and men, with the $15 \%$ rate. Eight percent of these users downloaded applications with health protection content such as hospital meeting, looking up doctor and/or pharmacy, $7 \%$ them accessed applications to measure body mass index, calories, and fitness. We should underline that these findings are consistent with $51 \%$ of our sample aged between 18-35 who take care of their health and 49\% of our sample aged between 36-45+ who are aware of chronic diseases.

(4) The majority of our sample were searching for their personal health condition-related information before going to a doctor (83\%), and information on pharmaceuticals (82\%), and treatment prescriptions information (80\%) after went to a doctor.

(5) Thirty seven percent of the participants affirmed that they use mobile health applications to be informed on health topics. Twenty eight percent of them to protect their health and $27 \%$ of them to create a healthy life.

These findings have stronger and consistent impact among our sample saying to look up health information on Internet with $95 \%$ ratio. We may suggest that to develop health promoting mobile applications will be a good investment opportunity in mobile health technologies market.

\footnotetext{
${ }^{3}$ Deloitte Report "mHealth in an mWorld: How mobile technology is transforming health care". Deloitte Center for Health Solutions. Available from http://www.deloitte.com/centerforhealthsolutions
} 


\section{Conclusion}

Digital technologies, including ubiquitous mobile devices, can play a key role in transforming health care system on-demand access to the medical records and powerful clinical decision support tools that empower patients to actively participate in their treatment plans. Mobile applications become more user-friendly and technologies expand their reach to remote populations.

In the present paper we explored some of the ICT in health uses and preferences of mobile health applications in Turkey.

Although we found very high levels of ICT use in our sample, limitations in our sample do restrict the generalisability of our findings. We conclude that it is imperative that researchers examine how health consumers use new technologies and target health promotion strategies according to user trends. For example, a very high number of our women and men participants used mobile smart phones and also applications when they were aged from 36 to $45+$. Because these people are aware of chronic disease threats and they are afraid of being sick. Yet, as discussed above, even the most popular of mobile applications is not used by all people in the same way. Women have varying levels of access to ICTs and they use them in diverse ways for different purposes. We can not assume that everyone has the same level of access to, or experience of, ICTs such as the Internet (O'Mara, 2012). Numerous factors, such as socioeconomic background, education, ethnicity, language, age, gender, communication preferences and digital literacy, health literacy have a significant impact on how the people use new health technologies. In addition to this, these findings lead to build efficient health promotion strategies based on effective health communication.

Hence, we recommend that mhealth interventions need to consider the importance of health literacy. However, for health services, our data highlight that new health and communication technologies are increasingly central tools to seek and manage information from lower SES to higher ones.

The lack of linguistic diversity in content was a limitation having an impact on the overall effectiveness of the mobile apps. Because our sample mentioned that $74 \%$ have the potential to use mHealth applications in future. Eighty five percent of our participants use only one mHealth application in Turkish. We found a significant negative correlation between the number of used mHealth application and the language. The language preference transformed from Turkish to English when the number of used mHealth application raised. We may suggest that there is a huge lack, and also a huge potential, of Turkish mHealth application in APP store.

Opportunities for developing new mHealth apps, content, products, and services are emerging daily. New fields of start-ups including highly personalized health care, population health, diagnostics, utilization management, consumer and provider incentivization, targeting specific populations for public health campaigns, and managing global and national epidemics, chronic diseases, transmitting and analyzing self data via censors will develop.

The industry and stakeholders with positive behavioral change, people engagement, prevention objectives in self healthcare and with extracting necessary and meaningful personal information will have a significant impact on people's health status and well-being.

\section{References}

Connelly, K. H., Faber, A. M., Rogers, Y., Siek, K. A., \& Toscos, T. (2006). Mobile applications to empower people to monitor their personal health. Retrieved from https://www.ipfw.edu/dotAsset/142360.pdf 
Downer, S. R., Meara, J. G., Da Costa A. C., \& Sethuraman, K. (2006). Sms text messaging improves outpatient attendance. Australian Health Review, 30, 389-396.

Ferrer-Roca, O., Cardenas, A., \& Diaz-Cardama, A. (2004). Mobile phone text messaging in the management diabetes. Journal of Telemdicine and Telecare, 10, 282-285.

Fisher, J., \& Clayton, M. (2012). Who gives a tweet: Assessing patients' interest in the use of social media for health care. Worldviews on Evidence-Based Nursing. Retrieved from http://www.ncbi.nlm.nih.gov/pubmed/2243273

Halpert, A., Dalton, C. B., Palsson, O., Morris, C., Hu, Y., Bangdiwala, S., Hankins, J., Norton, N., \& Drossman, D. A. (2008). Patient educational media preferences for information about irritable bowel syndrome (IBS). Digestive Diseases and Sciences, 53, p. 3184.

Information and Communication Technology Authorities (ICTA). Electronic communications market in Turkey. Market Data (2013 Q4).

IPSOS Media Ct. (2013). Our mobile planet Turkey, understanding the mobile consumer. May 2013.

Kim, H-S., Kim, N-C., \& Ahn, S-H. (2006). Impact of a nurse short message service intervention for patients with diabetes. Journal of Nursing Care Quality, 21, 266-271.

Klasnja, P., Consolvo, S., Mc Donald, D. W., Landay, J. A., \& Pratt, W. (2009). Using mobile \& personal sensing technologies to support health behaviour change in everyday life: Lessons learned. University of Washington, Settle, WA; Intel Research Seattle, Seattle, WA.

Leong, K. C., Chen, W. S., Leong, K. W., Mastura, I., Mimi, O., Sheikh, M. A., Zailinawati, A. H., Ng, C. J., Phua, K. L., \& Teng, C. L. (2006). The use of text messaging to improve attendance in primary care: A randomized controlled trial. Family Practice, 23, 699-705.

Levine, D. (2007). Using technology to promote youth sexual health. Mobile Persuasion. Persuasive Technology Lab, Standford University.

O’Mara, B. (2012). Social media, digital video and health promotion in a culturally and linguistically diverse Australia. Health Promotion International. doi: 10.1093/heapro/das014, 2012.

Puccio, J. A., Belzer, M., Olson, J., Martinez, M., Salata, C., Tucker, D., \& Tanaka, D. (2006). The use of cell phone reminder calls for assisting HIV-infected adolescents and young adults to adhere to highly active antiretroviral therapy: A pilot study. AIDS Patient Care \& STDs, 20, 438-444.

Rodgers, A., Corbett, T., Bramley, D., Riddell, T., Wills, M., Lin, R-B., \& Jones, M. (2005). Do you smoke after txt? Results of a randomized trial of smoking cessation using mobile phone text messaging. Tobacco Control, 14, p. 257.

Tate, D. F., Wing, R. R., \& Winett, R. A. (2001). Using internet technology to deliver a behavioral weight loss program. Proceedings of the American Marketing Association Conference 2001, Washington, DC.

Thackeray, R., Neiger, B. L., Hanson, C. L., \& McKenzie, J. F. (2008). Enhancing promotional strategies within social marketing programs: Use of Web 2.0 social media. Health Promotion Practice, 2008.

WHO. Mhealth new horizons for health through technologies. Mobile global observatory for eHealth series, 3, p. 5. 